\title{
The Potential Clinical and Economic Value of Primary Tumour Identification in Metastatic Cancer of Unknown Primary Tumour: A Population-Based Retrospective Matched Cohort Study
}

\author{
Malek B. Hannouf ${ }^{1,2} \cdot$ Eric Winquist $^{3} \cdot$ Salaheddin M. Mahmud $^{4}$.

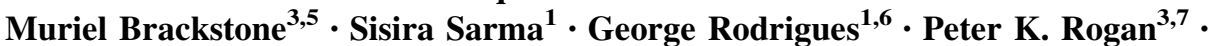 \\ Jeffrey S. Hoch ${ }^{8,9,10} \cdot$ Gregory S. Zaric $^{1,2}$
}

Published online: 6 September 2017

(C) The Author(s) 2017. This article is an open access publication

\begin{abstract}
Purpose Several genomic tests have recently been developed to identify the primary tumour in cancer of unknown primary tumour (CUP). However, the value of identifying the primary tumour in clinical practice for CUP patients remains questionable and difficult to prove in randomized trials.

Objective We aimed to assess the clinical and economic value of primary tumour identification in CUP using a retrospective matched cohort study.

Methods We used the Manitoba Cancer Registry to identify all patients initially diagnosed with metastatic cancer between 2002 and 2011. We defined patients as having CUP if their primary tumour was found 6 months or more after initial diagnosis or never found during the course of disease. Otherwise, we considered patients to have metastatic cancer from a known primary tumour (CKP). We linked all patients with Manitoba Health databases to
\end{abstract}

Electronic supplementary material The online version of this article (doi:10.1007/s41669-017-0051-2) contains supplementary material, which is available to authorized users.

Malek B. Hannouf

mbassamh@uwo.ca

1 Department of Epidemiology and Biostatistics, Schulich School of Medicine and Dentistry, Western University, 1255 Western Road, London, ON N6G 0N1, Canada

2 Ivey Business School, Western University, London, ON, Canada

3 Department of Oncology, Schulich School of Medicine and Dentistry, Western University, London, ON, Canada

4 Department of Community Health Sciences, College of Medicine, Faculty of Health Sciences, University of Manitoba, Winnipeg, MB, Canada estimate their direct healthcare costs using a phase-of-care approach. We used the propensity score matching technique to match each CUP patient with a CKP patient on clinicopathologic characteristics. We compared treatment patterns, overall survival (OS) and phase-specific healthcare costs between the two patient groups and assessed association with OS using Cox regression adjustment.

Results Of 5839 patients diagnosed with metastatic cancer, 395 had CUP (6.8\%); 1:1 matching created a matched group of 395 CKP patients. CUP patients were less likely to receive surgery, radiation, hormonal and targeted therapy and more likely to receive cytotoxic empiric chemotherapeutic agents. Having CUP was associated with reduced OS (hazard ratio [HR] 1.31; 95\% confidence interval 1.1-1.58), but this lost statistical significance with adjustment for treatment differences. CUP patients had a significant increase in the mean net cost of initial diagnostic workup before diagnosis and a significant reduction in the mean net cost of continuing cancer care.

Conclusion Identifying the primary tumour in CUP patients might enable the use of more effective therapies,

5 Department of Surgery, Schulich School of Medicine and Dentistry, Western University, London, ON, Canada

6 Department of Radiation Oncology, London Regional Cancer Program, London, Canada

7 Department of Biochemistry, Schulich School of Medicine and Dentistry, Western University, London, ON, Canada

8 Institute of Health Policy Management and Evaluation, University of Toronto, Toronto, ON, Canada

9 Li Ka Shing Knowledge Institute, St. Michael's Hospital, Toronto, ON, Canada

10 Department of Public Health Sciences, University of California, Davis, USA 
improve OS and allow more efficient allocation of healthcare resources.

\section{Key Points for Decision Makers}

Cancer of unknown primary tumour (CUP) is the fifth most common cancer type in patients presenting with metastatic cancer.

Compared with patients with metastatic cancer of known primary, patients with CUP receive fewer site-specific cancer treatments, have reduced overall survival, and use more healthcare resources for diagnostic workup but less healthcare resources for cancer care.

Identifying the primary tumour in CUP patients might enable the use of more precise anticancer therapy, improve overall survival and allow more efficient allocation of healthcare resources.

The use of accurate genomic tests to help identify the primary tumour in CUP might be clinically and economically warranted.

\section{Introduction}

Approximately $15 \%$ of all new cancers present with metastases at time of diagnosis [1]. Primary site determination for metastatic cancers is the starting point to define standard-of-care patient management. In approximately two-thirds of these cases, the primary tumour becomes apparent early during the course of the initial diagnostic workup [2]. The remaining metastatic cancers represent challenging cases as tumours of uncertain primary origin. For these cases, pathologists and oncologists often undertake a comprehensive diagnostic workup that may include cytogenetic studies, electron microscopy, endoscopies and immunohistochemical (IHC) analysis [2, 3]. Although clinical and pathologic investigations are recommended to be sign and symptom guided [2,3], they often require a longer hospital stay accompanied by painful and distressing investigations and may incur increased costs for the healthcare system $[4,5]$. However, a primary tumour site is identified in $<30 \%$ of these cases [6,7]. Consequently, over 6800 cases with histologically proven metastatic cancer of unknown primary origin (CUP) are diagnosed annually in Canada despite diagnostic assessment.

Although it is accepted that CUP is a heterogeneous collection of metastatic malignancies [6], there is no consensus on whether CUP is simply a group of metastatic tumours with undetected primary tumours or a distinct entity with specific genetic characteristics [6]. Clinically occult primary tumours have subsequently been anatomically detected at autopsy in about $75 \%$ of CUP patients [8]. In addition, approximately $7 \%$ of CUP patients have their latent primary (LP) tumour clinically detected months to years after initial diagnosis [9]. Most metastatic tumour biopsies from CUP patients are completely undifferentiated or poorly differentiated, with the majority being adenocarcinomas, but other histologies have also been diagnosed (e.g. lymphoma, melanoma, sarcoma) [1, 10].

In the absence of a specific primary tumour diagnosis, treatment guidelines vary [3]. When appropriate, CUP patients are typically treated with empirical cytotoxic chemotherapy regimens $[11,12]$. Despite this, CUP patients have a poor prognosis, with median survival typically ranging from 7 to 11 months [6].

In recent years, the emergence of gene expression tumour-profiling assays (messenger RNA [mRNA] or microRNA platforms) as adjuncts to current clinicopathologic evaluations has offered the potential to correctly identify the primary tumour in about $90 \%$ of patients with CUP [7, 13-26]. This breakthrough, coupled to the recent emergence of targeted drugs for patients with known primary tumour (CKP) fuelled hopes for a revamp of CUP management [27-29]. Although the clinicopathologic presentation of CUP patients may differ from that of patients with CKP, there does not seem to be a major disparity in the response rates and the outcomes between these two patient groups after the primary tumour is defined and tumour-specific therapies are administered [29]. However, the impact of primary tumour identification via gene expression profiling on clinical outcomes for patients with CUP remains uncertain and difficult to prove in randomized trials because of a combination of ethical, clinical and logistical considerations [29-31]. Additional clinical evidence is necessary to optimize a precision medicine strategy as a standard of care for patients with CUP. In addition, the impact of primary tumour identification on the cost of care throughout the course of metastatic disease will be an important element in the formulation of new provincial guidelines and reimbursement policies for CUP management and necessary to inform healthcare resource allocation and fiscal planning for the prospective precision medicine era of care for CUP.

In this study, we aimed to estimate the potential clinical and economic value of primary tumour identification in CUP using heath administrative databases from the Canadian province of Manitoba to carry out a propensity score matched-cohort analysis in which we evaluated differences in clinical and economic outcomes between patients with CUP and their matched counterparts with CKP. 


\section{Patients and Methods}

\subsection{Data Sources and Identification of Study Population}

The Manitoba Cancer Registry (MCR) and Provincial Pharmacy program at CancerCare Manitoba (CCMB) and Manitoba administrative databases held by Manitoba Health, including the Hospital Discharge Database, Physician Claims Database and the Drug Program Information Network (DPIN), served as the data sources for this analysis. A full description of these databases and their contents has been reported elsewhere [30-33].

We have previously used the MCR to identify a cohort of patients diagnosed initially with metastatic cancer during the period from 1 January 2002 to 31 December 2011 [34] and identify when these patients had their primary tumour diagnosed during the course of their disease. The cohort was limited to Manitoba residents who had undergone clinical and pathological diagnostic evaluation in Manitoba and with no history of other malignancy at initial diagnosis. A minimum of 2-year follow-up information from the time of initial diagnosis was available for each patient. Follow-up information included diagnosis of second primary, cancer treatments (e.g. surgical and therapeutic radiology procedures, systemic therapy and palliative care) and death. Full details regarding the identification of this metastatic patient population and the process by which we identified when primary tumours were diagnosed are reported elsewhere [34].

For this analysis, we used this metastatic patient population to identify all patients who had their metastatic disease histologically confirmed and survived at least 6 months following their initial cancer diagnosis. This 6-month window was used to ensure that patients would have had reasonable time during the early course of their metastatic disease to undergo all necessary clinical and pathological diagnostic evaluations (i.e. diagnostic workup) and had their primary tumour sites diagnosed [9]. We stratified patients by their primary tumour diagnostic status (CUP vs. CKP). We defined patients as having CUP if their primary tumour was initially unidentified (i.e. International Classification of Diseases for Oncology [ICDO] code C80.9) and either had their LP tumour diagnosed at least 6 months after their initial diagnosis or who never had their LP tumour diagnosed during the 2-year follow-up period. The 6-month window was used to ensure the identification of the LP tumour was not the result of extension in the initial diagnostic workup and is considered conservative compared with other attempts at identifying CUP using a 2-month window [9]. We defined patients as having CKP if their primary tumour was initially identified during the 6 months after their initial diagnosis and no change was detected in their primary tumour diagnostic status later during the 2-year follow-up period.

\subsection{Linkage with the Provincial Pharmacy Program Database at CCMB and Manitoba Administrative Databases}

We linked the two patient groups with the Provincial Pharmacy Program of CCMB and Manitoba Health's administrative databases to validate all cancer therapy data captured by the MCR; to collect additional information on types of radiotherapy and systematic therapy agents; to measure co-morbidity; and to estimate direct healthcare costs. To protect confidentiality, the linkage was performed with a scrambled unique health number using anonymized versions of these databases. Records of these databases for each patient in the study cohort were available between 3 years before and 2 years after initial cancer diagnosis.

\subsection{Comorbidity Index}

We determined co-morbidity from the Hospital Discharge Database and the Physician Claims Database through diagnoses that were recorded for each patient in the study cohort during all patient hospital stays and physician claims between 3 years before and 6 months after initial cancer diagnosis. We used co-morbid diagnoses coded using the Charlson Co-morbidity Index, excluding cancer diagnoses, which has been used elsewhere [32-34].

We also used the Physician Claims Database to collect information on diagnostic tests and procedures undertaken during the diagnostic workup (defined as the period from 6 months before to 6 months after initial cancer diagnosis) for all identified patients diagnosed with metastatic cancer. The diagnostic tests and procedures recorded in the Physician Claims Database include the following: endoscopies, diagnostic ultrasound scans, X-rays, computerized axial tomography (CT) scans, magnetic resonance imaging (MRI) scans, diagnostic isotope procedures, fine and core needle biopsies, pulmonary function tests, lumbar puncture (spinal tap), dermatoscopy, guaiac faecal occult blood test, gynaecological physical examination, Papanicolaou (Pap) test, and dilatation and curettage.

\subsection{Direct Healthcare Costs}

The direct costs of healthcare services comprised inpatient and 1-day procedure stays, physician and other healthcare provider services, prescription drugs and intravenous oncology drugs. The costs of these services and drugs are all publicly funded in Manitoba and recorded in the 
Hospital Discharge Database [35, 36], Physician Claims Database, DPIN database and Provincial Pharmacy Program of CCMB database. We determined the healthcare costs from all records of these databases for each patient in the study cohort between 3 years before and 2 years after initial cancer diagnosis. All costs are expressed in Canadian dollars (CAD), year 2016 values, using the Bank of Canada inflation calculator [37].

We estimated the costs of various service and drug categories using a phase-of-care approach [38-41]. Costs of care for patients in the cohort were divided into five clinically relevant phases of care: (1) usual care before the onset of signs of illness; (2) initial diagnostic workup before diagnosis; (3) continuing diagnostic workup after diagnosis and initial cancer care; (4) continuing cancer care; (5) last 6 months of life. The "usual care before the onset of signs of illness" phase consisted of the time between 3 years and 6 months before diagnosis. We defined the "initial diagnostic workup before diagnosis" phase as the 6 months before diagnosis and the "continuing diagnostic workup after diagnosis and initial cancer care" phase as the 6 months after diagnosis. Among the patients who died, the "last 6 months of life" phase was defined as the final 6 months of life. The "continuing cancer care" phase consisted of the time between the "continuing diagnostic workup after diagnosis and initial cancer care" and "last 6 months of life" phases. Because all patients included in our study cohort survived at least 6 months following their initial cancer diagnosis (i.e. by our inclusion criteria), we first allocated costs to the first two phases of care ("usual care before the onset of signs of illness" and "initial diagnostic workup before diagnosis) and the "last 6 months of life" phase. Then, we allocated costs to the "continuing diagnostic workup after diagnosis and initial cancer care" phase, and any remaining costs were allocated to the "continuing cancer care" phase. To determine whether costs incurred during the last 6 months of the 2-year follow-up period should be allocated to the "last 6 months of life" phase or to the "continuing cancer care" phase, it was necessary to collect from the MCR an additional 6 months of death information following the 2-year follow-up from the time of initial diagnosis.

\subsection{Statistical Analysis}

In our primary analysis, we used the propensity scorematching method to build comparable CUP and CKP groups in terms of observable characteristics [42-44]. Full details on propensity score matching are provided as Electronic Supplementary Material (ESM).

We constructed 2-year Kaplan-Meier curves to examine the cumulative probability of overall survival (OS) for the two patient groups. The curves were compared using the log-rank test statistic. We then used a Cox proportional hazards model to test the effect of the diagnostic status of primary tumour (CUP vs. CKP) on the 2-year OS by calculating the hazard ratio (HR) for death with associated 95\% confidence intervals (CIs) for CUP versus CKP. Receipt of cancer treatments (surgery [yes vs. no], radiotherapy [yes vs. no], systematic therapy [yes vs. no]), time to receive cancer treatments following initial diagnosis (number of months to surgery, radiotherapy and chemotherapy), receipt of biological targeted therapy (targeted biological therapy + chemotherapy vs. chemotherapy alone) were all tested for associations with OS and tested for their effect on the calculated HR for CUP versus CKP by adjusting the Cox proportional hazards model for these treatment covariates. We also examined whether the association of these treatment covariates with OS differed in CUP versus CKP by testing for interactions between treatment covariates and the diagnostic status of primary tumour (CUP vs. CKP).

In secondary analyses, we used three different methods to adjust for differences in baseline characteristics between the CUP group and all patients with CKP in the full cohort and examined the effect of diagnostic status of primary tumour (CUP vs. CKP) on the 2-year OS by calculating adjusted HRs and 95\% CIs for CUP versus CKP. First, we adjusted for the propensity score by including it as a continuous covariate in a Cox proportional hazards model. Second, we adjusted for all baseline characteristics by including them as covariates in a traditional multivariableadjusted Cox proportional hazards model. Third, we used a weighted Cox proportional hazards model, where the weight assigned for each patient was based on the stabilized inverse propensity score, as described elsewhere [43].

Within each phase of care and for each cost category, we calculated the cost per day for each patient in our cohort and the average cost per day separately for the CUP group, matched CKP group and all patients with CKP in the full cohort. We then estimated the average net cost per day for each phase of care following the "usual care before the onset of signs of illness" phase as the difference in average cost per day between the "usual care before the onset of signs of illness" phase and each of the following phases of care. We estimated the average net cost of an entire phase of care as the average net cost per day multiplied by the average number of days spent in that phase of care. We also calculated these cost estimates for the overall healthcare utilization by combining all cost categories.

\section{Results}

We identified 5839 patients who were initially diagnosed with metastatic cancer during the period from 1 January 2002 to 31 December 2011 and met our study inclusion criteria. Of those, 395 had CUP (6.8\%) and 5444 (93.2\%) 
had CKP (Table 1). Among those patients with CUP, 219 were women and 176 were men, accounting for $8.8 \%$ of all women and $5.3 \%$ of all men diagnosed with metastatic cancer, respectively (mean difference 3.5\%, 95\% CI 2.2-4.9; $p<0.0001$; Table 1). Among those patients with CUP, 169 (42.8\%) had their LP tumour diagnosed later, after 6 months from their initial diagnosis, and 226 (57.2\%) never had their LP tumour diagnosed during the 2 -year follow-up period. Table 2 shows the final classification of primary tumour site later in the course of metastatic disease for 395 patients initially diagnosed with CUP.

Prior to matching, patients with CUP presented with clinicopathological features distinct from those of their counterparts of all patients with CKP (Table 3). Using 1:1 matching on the estimated propensity score, we matched the CUP group of 395 patients with a CKP group of 395 patients. No CUP cases were dropped due to poor match quality. Table 3 shows the baseline patient and tumour characteristics of the matched CKP group as compared with the CUP group. As a result of matching, we eliminated differences between the two groups in age, sex, year of initial diagnosis, co-morbidity score, grade differentiation, histology and number and type of metastatic sites (Table 3).

The diagnostic tests and procedures undertaken during the diagnostic workup for the CUP group compared with the CKP matched group and all patients with CKP in our cohort are described in Table S1 in the ESM. During the diagnostic workup, compared with the matched CKP group, the CUP group were more likely to receive colonoscopy, gastroscopy and/or esophagogastroduodenoscopy, laryngoscopy and pharyngoscopy, laparotomy, panendoscopy (respiratory system); CT scans of the neck, abdomen and pelvis, and spine; MRI scans of the spine; ultrasound scans of the chest, abdomen and retro- peritoneum, female pelvis, miscellaneous scans (Doppler studies); thyroid biopsy; and mammography (Table S1). However, CUP patients were less likely to receive bronchoscopy, mediastinoscopy, proctosigmoidoscopy and pulmonary function tests than were the matched CKP group (Table $\mathrm{S} 1$ ). Other diagnostic tests and procedures were similar between the two patient groups (Table S1).

\subsection{Treatment Characteristics}

Compared with matched CKP patients, patients with CUP were less likely to have surgical resections (mean difference 18.5\%; 95\% CI 11.8-25; $p<0.0001)$, receive radiotherapy (mean difference $9.1 \%$; 95\% CI 2.5-15.7; $p=0.007$ ) and receive systemic therapy (mean difference 9.1\%; 95\% CI 2.2-16; $p=0.009$ ) (Table 4). Time to any of these therapies after initial diagnosis did not differ significantly between the CUP and matched CKP groups (Table 4). Among all patients who received systemic therapy, CUP patients were less likely than matched patients with CKP to receive hormone therapy (mean difference $4.5 \%$; 95\% CI 1.9-7.1; $p=0.002$ ) and biological targeted therapy (mean difference 13.9\%; 95\% CI $5.3-22.4 ; p=0.002$ ) but more likely to receive platinum drugs (mean difference 11.2\%; 95\% CI 1.4-20.9; $p=0.02$ ) and taxanes (mean difference 18\%; 95\% CI 8.2-27.7; $p=0.0003$ ) (Table 4). Table 4 shows the treatment characteristics of the CUP group, matched CKP group and all patients with CKP.

\subsection{Survival Outcomes}

The OS of patients with CUP was worse than that of matched CKP patients (2-year OS 41.7 vs. $50.4 \%$, $p=0.005$, Fig. 1; HR 1.31, 95\% CI 1.1-1.58, $p=0.005$,
Table 1 Primary tumour site of 5839 patients diagnosed with metastatic cancer by sex

\begin{tabular}{llll}
\hline Primary tumour sites & Men $(n=3344)$ & Women $(n=2495)$ & All $(n=5839)$ \\
\hline Gastrointestinal tumours & $960(28.7)$ & $613(24.6)$ & $1573(27)$ \\
Lung and pleural tumours & $598(17.9)$ & $603(24.2)$ & $1201(20.6)$ \\
Male genital system & $676(20.2)$ & - & $676(11.6)$ \\
Lymphoma tumours & $264(7.9)$ & $211(8.5)$ & $475(8.1)$ \\
Unknown primary tumour site & $176(5.3)$ & $219(8.8)$ & $395(6.8)$ \\
Head and neck tumours & $282(8.4)$ & $90(3.6)$ & $372(6.4)$ \\
Urological tumours & $248(7.4)$ & $92(3.7)$ & $340(5.8)$ \\
Breast tumours & $1(<0.1)$ & $281(11.3)$ & $282(4.8)$ \\
Gynaecologic tumours & - & $265(10.6)$ & $265(4.5)$ \\
Melanoma (skin) tumours & $60(1)$ & $26(1)$ & $86(1.5)$ \\
Endocrine tumours & $38(1.1)$ & $44(1.8)$ & $82(1.4)$ \\
Bone and soft tissue sarcoma tumours & $35(1)$ & $41(1.6)$ & $76(1.3)$ \\
Other known primary tumours & $6(0.2)$ & $10(0.4)$ & $16(0.3)$ \\
\hline
\end{tabular}

Data are presented as $n(\%)$ 
Table 2 Final classification of primary tumour later in the course of metastatic disease for 395 patients initially diagnosed with cancer of unknown primary tumour

\begin{tabular}{|c|c|c|}
\hline $\begin{array}{l}\text { Initial classification of primary tumour site } \\
\text { early in the course of metastatic disease }\end{array}$ & $\begin{array}{l}\text { Final classification of primary tumour site } \\
\text { later in the course of metastatic disease }\end{array}$ & Patients, $n(\%)$ \\
\hline \multirow[t]{17}{*}{ Unknown primary tumour $(n=395)$} & Unknown primary tumour & $226(57.2)$ \\
\hline & Ovary & $23(5.8)$ \\
\hline & Colon and rectum & $22(5.5)$ \\
\hline & Non-small cell lung & $22(5.5)$ \\
\hline & Non-Hodgkin’s lymphoma & $19(4.8)$ \\
\hline & Buccal cavity and pharynx & $14(3.5)$ \\
\hline & Pancreas & $12(3)$ \\
\hline & Bone and soft tissue sarcoma & $11(2.8)$ \\
\hline & $\begin{array}{l}\text { Stomach, small intestine, hepatocellular liver, } \\
\text { and other digestive system }\end{array}$ & $9(2.3)$ \\
\hline & Melanoma (skin) & $7(1.8)$ \\
\hline & Prostate and testicular germ cell & $7(1.8)$ \\
\hline & Kidney, renal pelvis and ureter & $6(1.5)$ \\
\hline & Small cell lung and other lung & $6(1.5)$ \\
\hline & Thyroid and other endocrine system & $4(1)$ \\
\hline & Breast & $3(0.75)$ \\
\hline & Other female genital system & $3(0.75)$ \\
\hline & Other ill-defined sites & $1(0.25)$ \\
\hline
\end{tabular}

Table 5). In a Cox proportional hazard regression analysis, having a CUP compared with CKP became a non-significant independent predictor of OS when controlling for use of surgery, radiotherapy and systematic therapy (Table 5). In this analysis, receipt of surgical resection and systemic therapy were significant independent predictors of OS (Table 5). No interactions between treatments and primary tumour status (CUP vs. CKP) were identified.

In subgroup analysis that included only patients treated with systematic therapy from the CUP and matched CKP groups and controlled for use of surgery and radiotherapy, receipt of a biological targeted therapy was associated with survival advantage (HR $0.52 ; 95 \%$ CI $0.31-0.88$; $p=0.01)$. The type of chemotherapeutic agents received (platinum with or without taxanes vs. other chemotherapeutic combinations) and receipt of hormone therapy were not independent significant predictors of OS. No interactions between these therapies and primary tumour status (CUP vs. CKP) were identified.

Unadjusted and adjusted Cox proportional hazard regression analyses that compared the OS of the CUP group $(n=395)$ with that of all patients with CKP $(n=5444)$ revealed similar results (Fig. 1; Table 5).

\subsection{Direct Costs of Healthcare Utilization}

The estimated mean net total costs for the CUP group and matched CKP group were \$CAN38,900 and \$CAD38,850, respectively. The mean net costs in the "initial diagnostic workup before diagnosis" phase, "continuing diagnostic workup after diagnosis and initial cancer care" phase, "continuing cancer care" phase, and "last 6 months of life" phase accounted for 20.2, 46.5, 14.8 and $18.5 \%$ of the total mean net cost for the CUP group, respectively, and for $8.7,49.5,26$ and $16 \%$ of the total mean net cost for the matched CKP group, respectively. Figure 2 depicts the mean cost per day within each phase of care and the mean net cost of an entire phase of care by cost category for the CUP group, matched CKP group and all patients with CKP in the full cohort.

Within the "initial diagnostic workup before diagnosis" phase, compared with matched CKP patients, CUP patients had a significant increase in the mean net cost of inpatient and 1-day procedure stays (mean difference \$CAD2960; 95\% CI 650-5270), a significant increase in the mean net cost of physician and other healthcare provider services (mean difference \$CAD1425; 95\% CI 1008-1840), and a significant increase in the mean net cost of prescription drugs (mean difference \$CAD241; 95\% CI 75-406), accounting for a significant increase in the mean net cost of overall healthcare utilization within this phase of care (mean difference \$CAD4622; 95\% CI 1730-7520) (Fig. 2).

Within the "continuing diagnostic workup after diagnosis and initial cancer care" phase, compared with matched CKP patients, CUP patients had a non-significant 
Table 3 Baseline patient and tumour characteristics of 5839 patients diagnosed with metastatic cancer by diagnostic status of their primary tumours

\begin{tabular}{|c|c|c|c|c|c|}
\hline Characteristic & $\begin{array}{l}\text { Patients with } \\
\text { CUP } \\
(n=395)\end{array}$ & $\begin{array}{l}\text { Patients with } \\
\text { CKP } \\
(n=5444)\end{array}$ & $p$ value $^{\mathrm{a}}$ & $\begin{array}{l}\text { Matched patients } \\
\text { with CKP } \\
(n=395)^{\mathrm{b}}\end{array}$ & $p$ value $^{\mathrm{c}}$ \\
\hline Age at initial diagnosis, years & $\begin{array}{c}65.3 \pm 13.9 \\
(24-96)\end{array}$ & $\begin{array}{c}64.3 \pm 13.1 \\
(19-103)\end{array}$ & 0.14 & $\begin{array}{r}65 \pm 13.9 \\
(20-100)\end{array}$ & 0.9 \\
\hline \multicolumn{6}{|l|}{ Year of initial diagnosis } \\
\hline $2002-2003$ & $85(21.5)$ & $949(17.4)$ & \multirow[t]{5}{*}{0.06} & $84(21.3)$ & \multirow[t]{5}{*}{0.9} \\
\hline 2004-2005 & $75(19)$ & $1225(22.5)$ & & $71(18)$ & \\
\hline $2006-2007$ & $85(21.5)$ & $1185(21.8)$ & & $84(21.3)$ & \\
\hline 2008-2009 & $91(23)$ & $1104(20.3)$ & & $94(23.8)$ & \\
\hline 2010-2011 & $59(15)$ & $981(18)$ & & $62(15.7)$ & \\
\hline \multicolumn{6}{|l|}{ Sex } \\
\hline Men & $176(44.6)$ & $3168(58.2)$ & \multirow[t]{2}{*}{$<0.0001$} & $175(44.4)$ & \multirow[t]{2}{*}{0.9} \\
\hline Women & $219(55.4)$ & $2276(41.8)$ & & $220(55.6)$ & \\
\hline \multicolumn{6}{|l|}{ Grade differentiation or cell indicator } \\
\hline Well differentiated & $7(1.8)$ & $170(5)$ & \multirow[t]{5}{*}{$<0.0001$} & $9(2.2)$ & \multirow[t]{5}{*}{0.9} \\
\hline Moderately differentiated & $20(5)$ & $1259(37.2)$ & & $21(5.3)$ & \\
\hline Poorly differentiated & $65(16.5)$ & $1448(42.7)$ & & $63(15.9)$ & \\
\hline Undifferentiated & $286(72.4)$ & $2209(40.6)$ & & $287(72.6)$ & \\
\hline $\mathrm{T}$ cell/B cell & $17(4.3)$ & $358(6.6)$ & & $15(3.8)$ & \\
\hline \multicolumn{6}{|l|}{ Histology } \\
\hline Adenocarcinomas & $157(39.7)$ & $2645(48.6)$ & \multirow[t]{9}{*}{$<0.0001$} & $157(39.7)$ & \multirow[t]{9}{*}{0.9} \\
\hline Squamous cell carcinoma & $49(12.4)$ & $557(10.2)$ & & $51(12.9)$ & \\
\hline Mucinous and serous & $26(6.6)$ & $235(4.3)$ & & $27(6.8)$ & \\
\hline Undifferentiated carcinoma & $114(28.8)$ & $895(16.4)$ & & $112(28.3)$ & \\
\hline Melanoma & $11(2.8)$ & $96(1.8)$ & & $10(2.5)$ & \\
\hline Complex mixed and stromal & $5(1.3)$ & $24(0.4)$ & & $6(1.5)$ & \\
\hline $\begin{array}{l}\text { Transitional cell, ductal lobular medullary, complex epithelial, } \\
\text { soft tissue sarcomas, myomatous, germ cell, miscellaneous bone, } \\
\text { nerve sheath }\end{array}$ & $14(3.5)$ & $473(8.7)$ & & $13(3.3)$ & \\
\hline Hodgkin's lymphoma, NHLs, other lymphoma & $19(4.8)$ & $475(8.7)$ & & $19(4.8)$ & \\
\hline Others & 0 & $44(0.8)$ & & 0 & \\
\hline \multicolumn{6}{|l|}{ Number of metastatic sites } \\
\hline 1 & $215(54.4)$ & 2014 (36.9) & \multirow[t]{3}{*}{$<0.0001$} & $214(54.2)$ & \multirow[t]{3}{*}{0.9} \\
\hline 2 & $105(26.6)$ & $1702(31.3)$ & & $105(26.6)$ & \\
\hline$\geq 3$ & 75 (19) & $1728(31.7)$ & & $76(19.3)$ & \\
\hline \multicolumn{6}{|l|}{ Metastatic sites, frequency $(\%)$} \\
\hline Digestive system & $196(32.2)$ & $1859(21)$ & \multirow[t]{9}{*}{$<0.0001$} & $193(31.7)$ & \multirow[t]{9}{*}{0.7} \\
\hline Respiratory system & $96(15.8)$ & $1478(16.7)$ & & $102(16.8)$ & \\
\hline Bones and joints & $68(11.2)$ & 1207 (13.7) & & $67(11)$ & \\
\hline Brain and other nervous system & $24(3.9)$ & $509(5.8)$ & & $25(4.1)$ & \\
\hline Soft tissue (including heart) & $19(3.1)$ & $128(1.4)$ & & $18(3)$ & \\
\hline Skin & $11(1.8)$ & $59(0.7)$ & & $9(1.5)$ & \\
\hline Lymph nodes & $142(23.3)$ & $2887(32.7)$ & & $143(23.5)$ & \\
\hline Ill defined & $25(4.1)$ & $349(3.9)$ & & $26(4.3)$ & \\
\hline $\begin{array}{l}\text { Others, including buccal cavity and pharynx, breast, female } \\
\text { genital system, male genital system, urinary system, } \\
\text { hematopoietic and reticuloendothelial systems, endocrine system, } \\
\text { and eye and adnexa }\end{array}$ & $28(4.6)$ & $356(4)$ & & $25(4.1)$ & \\
\hline With second primary tumour & $18(4.6)$ & $266(4.9)$ & 0.7 & $18(4.6)$ & 1 \\
\hline
\end{tabular}


Table 3 continued

\begin{tabular}{|c|c|c|c|c|c|}
\hline Characteristic & $\begin{array}{l}\text { Patients with } \\
\text { CUP } \\
(n=395)\end{array}$ & $\begin{array}{l}\text { Patients with } \\
\text { CKP } \\
(n=5444)\end{array}$ & $p$ value $^{\mathrm{a}}$ & $\begin{array}{l}\text { Matched patients } \\
\text { with CKP } \\
(n=395)^{\mathrm{b}}\end{array}$ & $p$ value $^{\mathrm{c}}$ \\
\hline Charlson co-morbidity score ${ }^{\mathrm{d}}$ & $\begin{array}{l}0.3 \pm 0.7 \\
\quad(0-6)\end{array}$ & $\begin{array}{l}0.29 \pm 0.72 \\
\quad(0-12)\end{array}$ & 0.8 & $0.3 \pm 0.6(0-6)$ & 0.9 \\
\hline$>0$ & $92(23.3)$ & $1194(21.9)$ & 0.5 & $93(23.5)$ & 0.9 \\
\hline 0 & 303 & 4250 & & 305 & \\
\hline 1 & 75 & 941 & & 77 & \\
\hline$\geq 2$ & 17 & 253 & & 16 & \\
\hline
\end{tabular}

Data are presented as mean \pm standard deviation (range) or number of patients $(\%)$ unless otherwise indicated

CKP cancer from a known primary tumour, CUP cancer of unknown primary tumour, NHLs non-Hodgkin's lymphomas

${ }^{a}$ Patients with CUP $(n=395)$ vs. all CKP $(n=5444)$ using Fisher's exact or Chi squared tests. All statistical tests were two sided, and results were considered significant at the 5\% critical level. Statistical analysis was performed using SAS, version 9.3 (Cary, NC, USA)

b Patients were matched on the estimated propensity score

c Patients with CUP $(n=395)$ vs. matched CKP $(n=5444)$ using Fisher's exact or Chi squared tests

${ }^{\mathrm{d}}$ Co-morbid diagnoses were considered present if they were found during 1 year before and 6 months after the initial diagnosis with cancer

difference in the mean net cost of inpatient and 1-day procedure stays and physician and other healthcare provider services but had a significant decrease in the mean net cost of intravenous oncology drugs (mean difference \$CAD870; 95\% CI 255-1480) and a significant decrease in the mean net cost of prescription drugs (mean difference $\$ C A D 340 ; 95 \%$ CI 84-595), accounting for a non-significant difference in the mean net cost of overall healthcare utilization within this phase of care (Fig. 2).

Within the "continuing cancer care" phase, compared with matched CKP patients, CUP patients had a non-significant difference in the mean net cost of inpatient and 1-day procedure stays, a significant decrease in the mean net cost of physician and other healthcare provider services (mean difference \$CAD580; 95\% CI 290-875), a significant decrease in the mean net cost of prescription drugs (mean difference \$CAD450; 95\% CI 135-760), and a significant decrease in the mean net cost of intravenous oncology drugs (mean difference \$CAD1912; 95\% CI 1090-2740), accounting for a significant decrease in the mean net cost of overall healthcare utilization within this phase of care (mean difference \$CAD4390; 95\% CI 1100-7680) (Fig. 2).

Within the "last 6 months of life" phase, compared with matched CKP patients, CUP patients had a nonsignificant difference in the mean net cost of inpatient and 1-day procedure stays, physician and other healthcare provider services and intravenous oncology drugs and a significant increase in the mean net cost of prescription drugs (mean difference \$CAD477; 95\% CI 320-610), accounting for a non-significant difference in the mean net cost of overall healthcare utilization within this phase of care (Fig. 2).
Cost analyses that compared the CUP group with all patients with CKP $(n=5444)$ across all cost categories and phases of care revealed similar results (Fig. 2).

\section{Discussion}

Through a population-based analysis, we found CUP to be the fifth most common $(6.8 \%)$ cancer type diagnosed in patients presenting with metastatic cancer and to be more common in women $(8.8 \%)$ than in men $(5.3 \%)$. The most common histological subtypes found in CUP were adenocarcinoma $(40 \%)$, undifferentiated carcinoma $(29 \%)$ and squamous carcinoma (12\%). Although CUP patients appeared to undergo more diagnostic tests and procedures during the diagnostic workup than their matched CKP counterparts, patients with CUP did not have their LP tumour site identified, except in $42 \%$ of patients where it was discovered at a later phase of care (i.e. after 6 months from initial cancer diagnosis). The percentage of LP tumour detection later in the course of the disease in our CUP group was higher than previously reported estimates [9], which was likely due to our conservative inclusion criteria of at least 6-month survival.

Having CUP appears to be associated with less frequent use of surgical interventions, radiation and hormonal and biological targeted therapy as well as greater use of nonselective cytotoxic chemotherapeutic drugs such as platinum drugs and taxanes. There was a significant decrease in the mean net cost of overall healthcare utilization, including the cost of intravenous oncology drugs during the "continuing cancer care" phase. Less exposure to surgery and biological targeted therapy were independently 
Table 4 Treatments of 5839 patients diagnosed with metastatic cancer by diagnostic status of their primary tumours

\begin{tabular}{|c|c|c|c|c|c|}
\hline Characteristic & $\begin{array}{l}\text { Patients with CUP } \\
(n=395)\end{array}$ & $\begin{array}{l}\text { Patients with CKP } \\
(n=5444)\end{array}$ & $p$ value $^{\mathrm{a}}$ & $\begin{array}{l}\text { Matched patients with } \\
\text { CKP }(n=395)^{\mathrm{b}}\end{array}$ & $p$ value $^{\mathrm{c}}$ \\
\hline With surgical resection & $112(28.3)$ & $2673(49.1)$ & $<0.0001$ & $185(46.8)$ & $<0.0001$ \\
\hline \multicolumn{6}{|l|}{$\begin{array}{l}\text { Months between initial cancer diagnosis and } \\
\text { surgical resection }\end{array}$} \\
\hline Mean \pm SD (range) & $1.5 \pm 3(0-20.5)$ & $1.7 \pm 3.1(0-24)$ & 0.6 & $1.2 \pm 1.9(0-15.8)$ & 0.3 \\
\hline$\geq 0$ to $<3$ & 91 & 2222 & & 162 & \\
\hline$\geq 3$ to $<6$ & 13 & 256 & & 17 & \\
\hline$\geq 6$ to $<12$ & 6 & 134 & & 5 & \\
\hline$\geq 12$ to $<24$ & 2 & 61 & & 1 & \\
\hline With radiotherapy & $117(29.6)$ & $1968(36.1)$ & 0.009 & $153(38.7)$ & 0.006 \\
\hline \multicolumn{6}{|l|}{$\begin{array}{l}\text { Months between initial cancer diagnosis and } \\
\text { start of radiotherapy }\end{array}$} \\
\hline Mean \pm SD (range) & $4.1 \pm 4.7(0-23.7)$ & $5.2 \pm 5.4(0-24)$ & 0.01 & $4.6 \pm 4.7(0-22.9)$ & 0.34 \\
\hline$\geq 0$ to $<3$ & 72 & 955 & & 78 & \\
\hline$\geq 3$ to $<6$ & 17 & 402 & & 30 & \\
\hline$\geq 6$ to $<12$ & 20 & 379 & & 32 & \\
\hline$\geq 12$ to $<24$ & 8 & 232 & & 13 & \\
\hline \multicolumn{6}{|l|}{ Type of radiotherapy } \\
\hline Teletherapy & $117(100)$ & $1775(90.2)$ & 0.001 & $132(86.3)$ & $<0.0001$ \\
\hline Brachytherapy & 0 & $18(0.9)$ & & $4(2.6)$ & \\
\hline Teletherapy + brachytherapy & 0 & $40(2)$ & & $3(2)$ & \\
\hline Other types & 0 & $14(6.9)$ & & $14(9.1)$ & \\
\hline With systemic therapy & $207(52.4)$ & $3528(64.8)$ & $<0.0001$ & $243(61.5)$ & 0.009 \\
\hline \multicolumn{6}{|l|}{ Type of systemic therapy received } \\
\hline $\begin{array}{l}\text { Only CTX with or without biological targeted } \\
\text { therapy }\end{array}$ & $207(100)$ & $3298(93.5)$ & 0.0001 & $232(95.5)$ & 0.002 \\
\hline $\begin{array}{l}\mathrm{HT}^{\mathrm{d}}+\mathrm{CTX} \text { with or without biological } \\
\text { targeted therapy }\end{array}$ & 0 & $230(6.5)$ & 0.0001 & $11(4.5)$ & 0.002 \\
\hline \multicolumn{6}{|l|}{$\begin{array}{l}\text { Months between initial cancer diagnosis and } \\
\text { start of radiotherapy }\end{array}$} \\
\hline Mean \pm SD (range) & $3 \pm 3.6(0-22.9)$ & $3.4 \pm 3.8(0-23.8)$ & 0.1 & $3 \pm 3.2(0-18.3)$ & 0.8 \\
\hline$\geq 0$ to $<3$ & 146 & 2261 & & 162 & \\
\hline$\geq 3$ to $<6$ & 36 & 791 & & 58 & \\
\hline$\geq 6$ to $<12$ & 16 & 295 & & 11 & \\
\hline$\geq 12$ to $<24$ & 9 & 181 & & 12 & \\
\hline $\begin{array}{l}\text { With information about CTX and biologic } \\
\text { therapy agents received }\end{array}$ & $151(72.9)$ & $2613(74)$ & 0.2 & $180(74)$ & 0.1 \\
\hline With biological targeted therapy & $21(13.9)$ & $522(20)$ & 0.06 & $50(27.8)$ & 0.002 \\
\hline \multicolumn{6}{|l|}{ Type of CTX agents received } \\
\hline Platinum drugs ${ }^{\mathrm{e}}$ & $115(76.2)$ & $1528(58.5)$ & $<0.0001$ & $117(65)$ & 0.02 \\
\hline Alkylating agents & $3(2)$ & $44(1.7)$ & & $1(0.6)$ & 0.3 \\
\hline Antimetabolites ${ }^{\mathrm{f}}$ & $78(51.7)$ & $1316(50.4)$ & 0.7 & $88(48.9)$ & 0.6 \\
\hline Anthracyclines ${ }^{\mathrm{g}}$ & $21(13.9)$ & $420(16.1)$ & 0.4 & $20(11.1)$ & 0.4 \\
\hline Topoisomerase inhibitors ${ }^{\mathrm{h}}$ & $41(27.2)$ & $793(30.3)$ & 0.4 & $60(33.3)$ & 0.2 \\
\hline Taxanes ${ }^{\mathrm{i}}$ & $59(39.1)$ & $467(17.9)$ & $<0.0001$ & $38(21.1)$ & 0.0003 \\
\hline Vinca alkaloids & $1(0.7)$ & $175(6.7)$ & 0.003 & $7(3.9)$ & 0.06 \\
\hline Corticosteroids & $4(2.6)$ & $16(0.6)$ & 0.004 & $1(0.6)$ & 0.2 \\
\hline Other anti-tumour antibiotics ${ }^{j}$ & $3(2)$ & $72(2.8)$ & 0.6 & $1(0.6)$ & 0.3 \\
\hline $\begin{array}{l}\text { Type of biological targeted therapy agents } \\
\text { received }\end{array}$ & & & & & \\
\hline
\end{tabular}


Table 4 continued

\begin{tabular}{|c|c|c|c|c|c|}
\hline Characteristic & $\begin{array}{l}\text { Patients with CUP } \\
(n=395)\end{array}$ & $\begin{array}{l}\text { Patients with CKP } \\
(n=5444)\end{array}$ & $p$ value $^{\mathrm{a}}$ & $\begin{array}{l}\text { Matched patients with } \\
\text { CKP }(n=395)^{\mathrm{b}}\end{array}$ & $p$ value $^{\mathrm{c}}$ \\
\hline Bevacizumab & $4(2.6)$ & $153(5.9)$ & 0.09 & $20(11.1)$ & 0.003 \\
\hline Rituximab & $12(7.9)$ & $295(11.3)$ & 0.2 & $22(12.2)$ & 0.2 \\
\hline Cetuximab & $2(1.3)$ & $26(1)$ & 0.6 & $4(2.2)$ & 0.5 \\
\hline Interferon-alfa- $2 b$ & $2(1.3)$ & $14(0.5)$ & 0.2 & $2(1.2)$ & 0.6 \\
\hline Panitumumab & $1(0.6)$ & $18(0.7)$ & 0.9 & 0 & 0.4 \\
\hline Bortezomib & 0 & $7(0.3)$ & 0.9 & 0 & \\
\hline Other targeted therapy & $1(0.6)$ & $21(0.8)$ & 0.9 & $4(2.2)$ & 0.3 \\
\hline $\begin{array}{l}\text { With support drugs to control CTX-associated } \\
\text { side effects or conditions }\end{array}$ & $99(65.6)$ & $1580(60.5)$ & 0.2 & $114(63.3)$ & 0.7 \\
\hline
\end{tabular}

Data are presented as mean \pm SD (range) or number of patients $(\%)$ unless otherwise indicated

$C K P$ cancer from a known primary tumour, $C T X$ chemotherapy, $C U P$ cancer of unknown primary tumour, $S D$ standard deviation

${ }^{a}$ Patients with unknown primary $(n=395)$ vs. all known primary $(n=5444)$ using Fisher's exact or Chi square. All statistical tests were two sided and results were considered significant at the 5\% critical level. Statistical analysis was performed using SAS, version 9.3 (Cary, NC)

b Patients were matched on the estimated propensity score

c Patients with unknown primary $(n=395)$ vs. matched known primary $(n=5444)$ using Fisher's exact or Chi square

d Hormone therapy included abiraterone, buserelin, leuprolide, letrozole, zoladex, and bicalutamide

e Platinum drugs included carboplatin, cisplatin and oxaliplatin

f Antimetabolites included capecitabine, gemcitabine, 5-fluorouracil, and raltitrexed

g Anthracyclines included doxorubicin and epirubicin

$\mathrm{h}$ Topoisomerase inhibitors included etoposide and irinotecan

i Taxanes included paclitaxel and docetaxel

${ }^{\mathrm{j}}$ Other agents included dexamethasone, vincristine, streptozocin, cyclophosphamide, and mitomycin

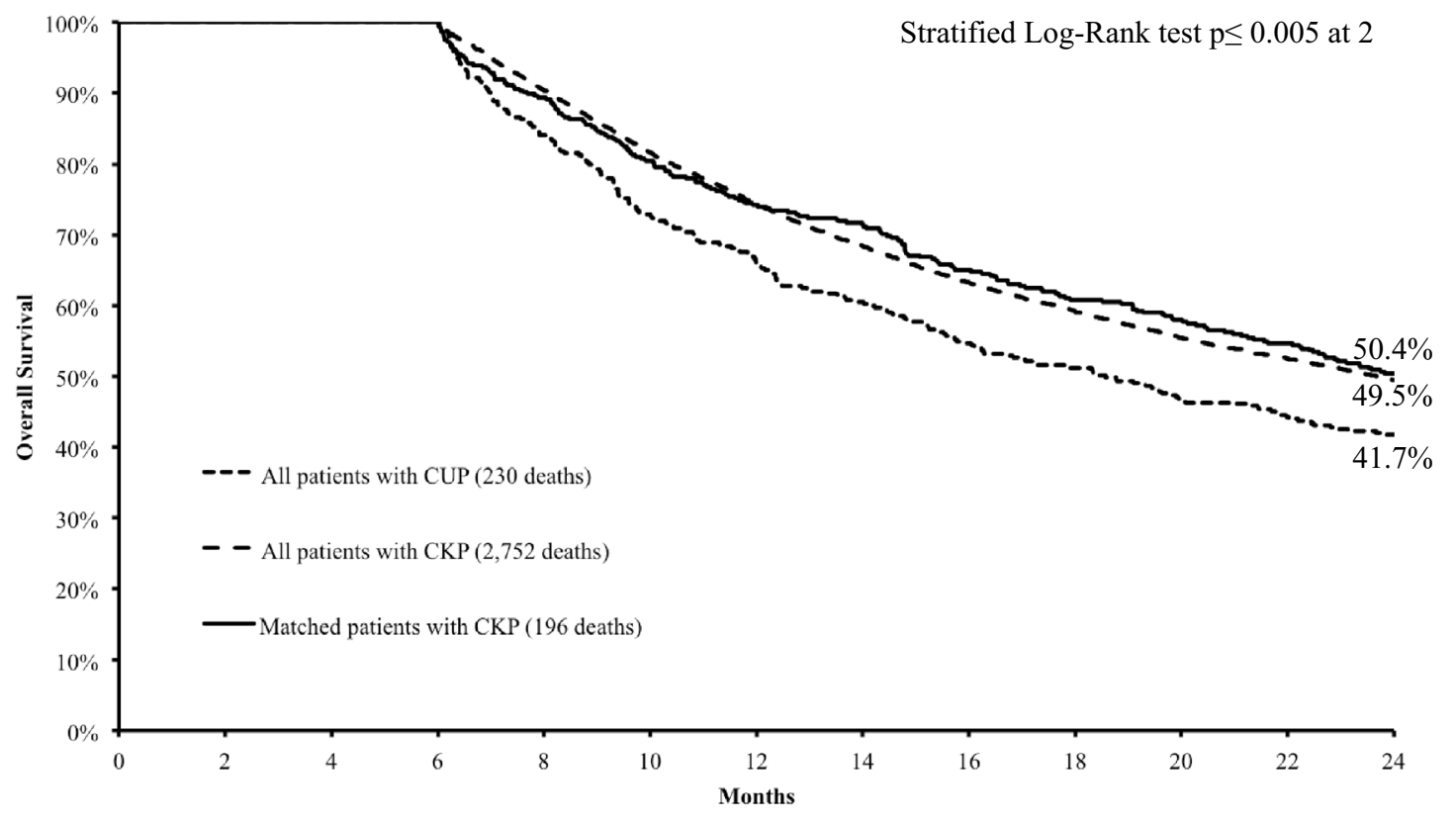

Number of patients at risk

\begin{tabular}{|c|c|c|c|c|}
\hline 395 & 395 & 262 & 202 & 165 \\
\hline 395 & 395 & 293 & 240 & 199 \\
\hline$--5,444$ & 5,444 & 4,049 & 3,221 & 2,692 \\
\hline
\end{tabular}

Fig. 1 Overall survival analyses comparing patients with cancer of unknown primary tumour (CUP) with patients with cancer from a known primary tumour (CKP) 


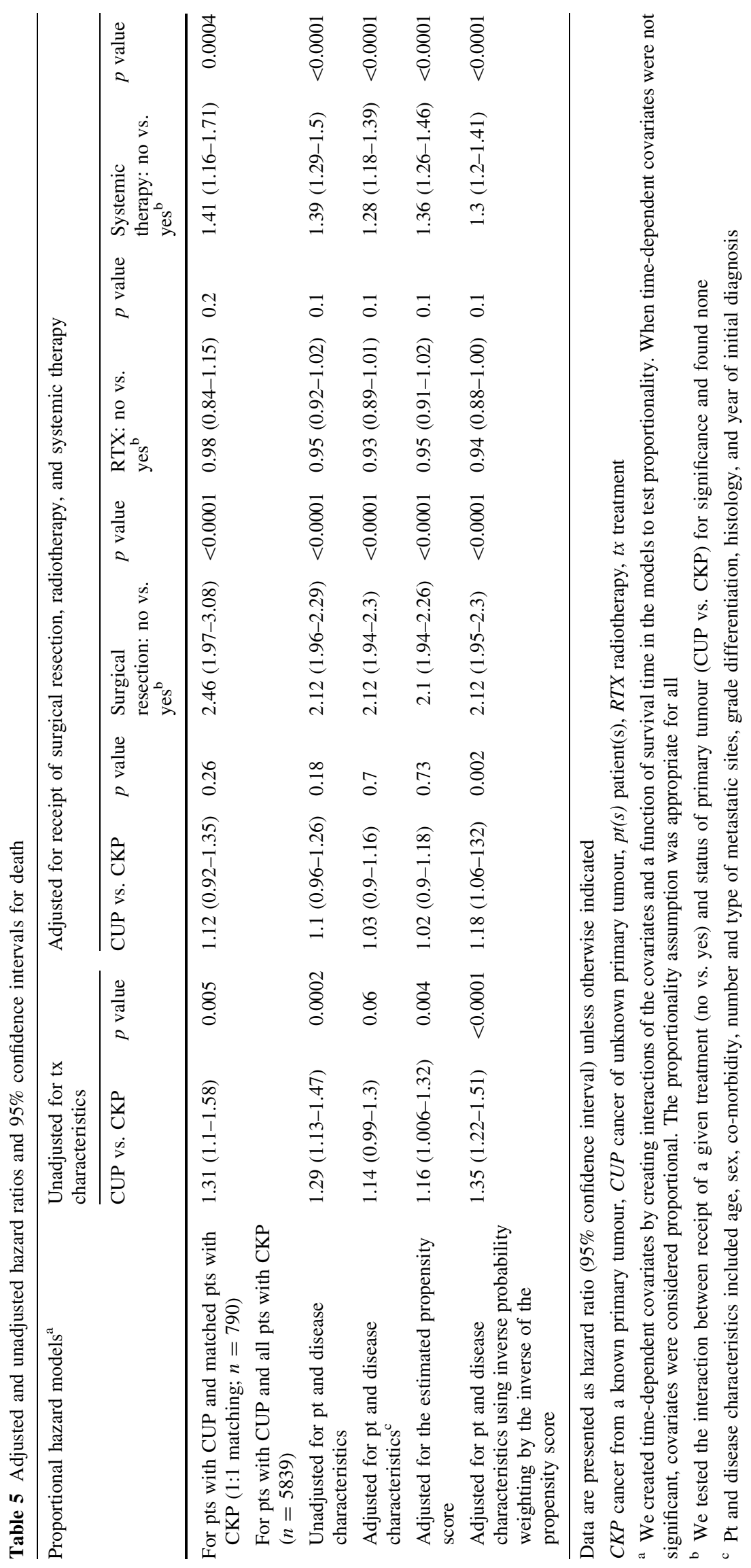


Inpatient and one day procedure stays*

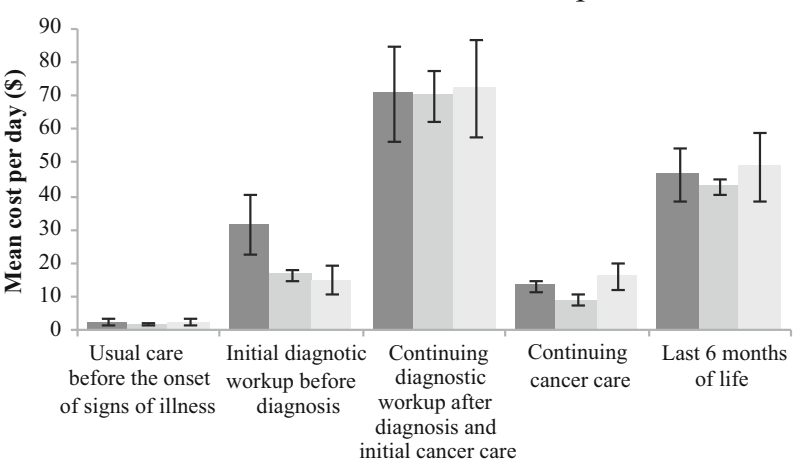

Phase of care

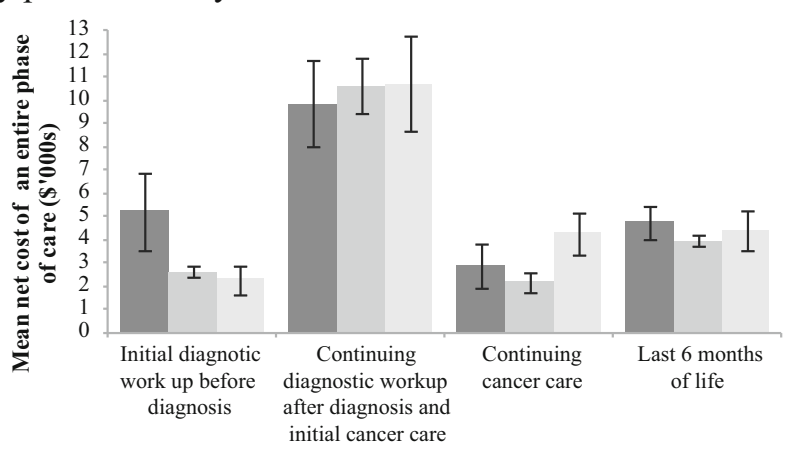

Phase of care

Physician and other healthcare provider' services

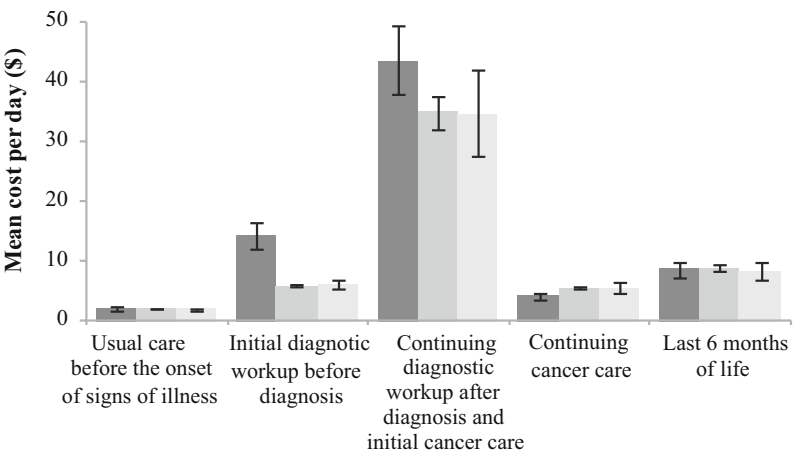

Phase of care

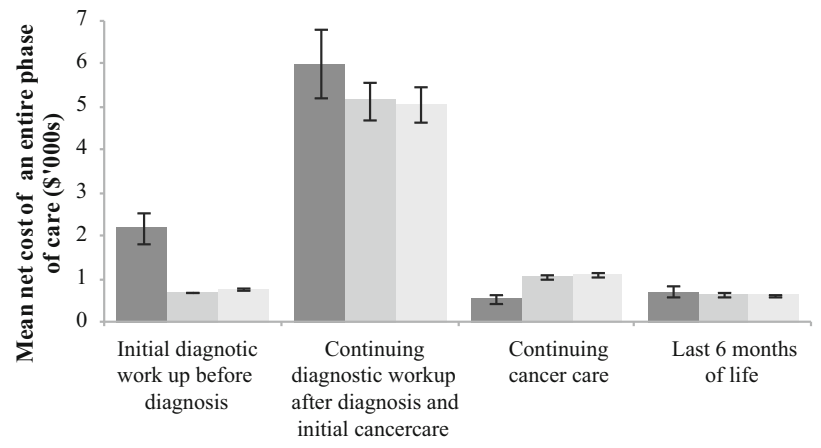

Phase of care

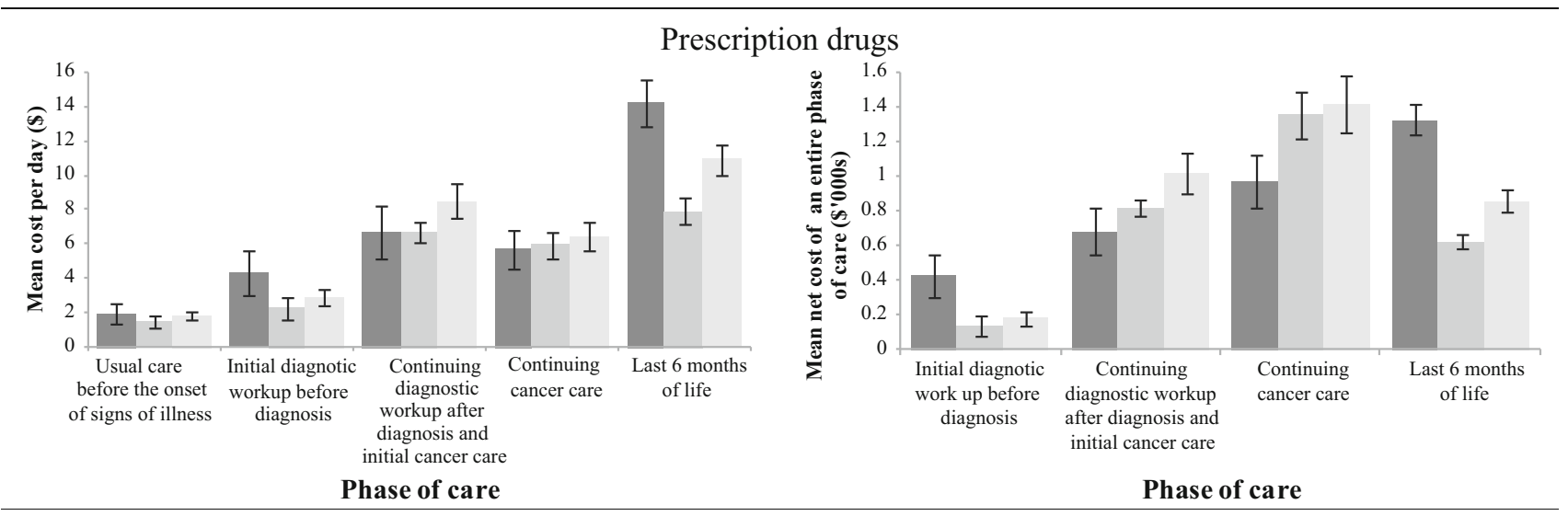

Fig. 2 Cost of healthcare utilization for 5839 patients diagnosed with metastatic cancer by diagnostic status of their primary tumour and cost category and phase of care. Mean net cost of an entire phase of care $=$ (mean cost per day in that phase of care - mean cost per day in the "usual care before the onset of signs of illness" phase) $\times$ average number of days spent in that phase of care. Since all patients included in our study survived at least 6 months following their initial cancer diagnosis, the three patient groups spent 2.5 years in the "usual care before the onset of signs of illness" phase and 6 months in the "initial diagnostic workup before diagnosis" phase. In the "continuing diagnostic workup after diagnosis and initial cancer care" phase, CUP patients, matched CKP patients and all patients with CKP spent an average of 144 days, 154 and 156 days, respectively. In the "continuing cancer care" phase, CUP patients, matched CKP patients and all patients with CKP spent an average of 260, 310 and 301 days, respectively. In the "last 6 months of life" phase, CUP patients, matched CKP patients and all patients with CKP spent an average of 108, 95 and 97 days, respectively. Asterisk We used the resource intensity weights [36, 37] recorded for inpatient stays and day procedure group weights [36, 37] recorded for day procedure stays to reflect the resources consumed during hospital contacts. We converted these weights into Canadian dollars using a multiplier known as the cost per weighted case [36, 37]. CKP cancer from a known primary tumour, $C U P$ cancer of unknown primary tumour 


\section{Intravenous oncology drugs}
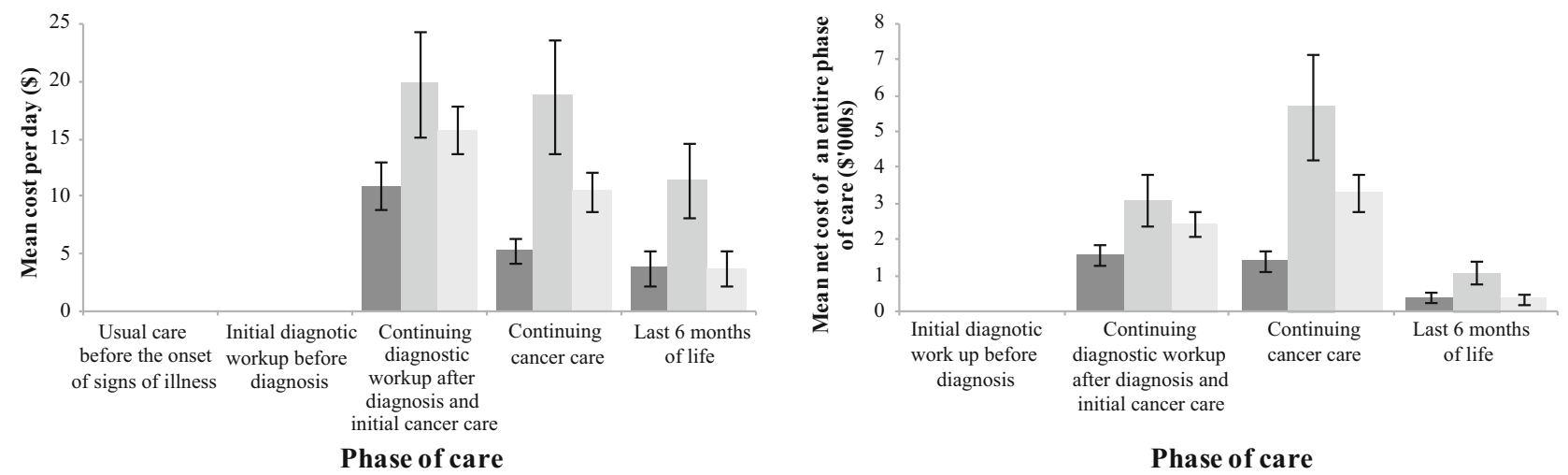

Overall health care utilization
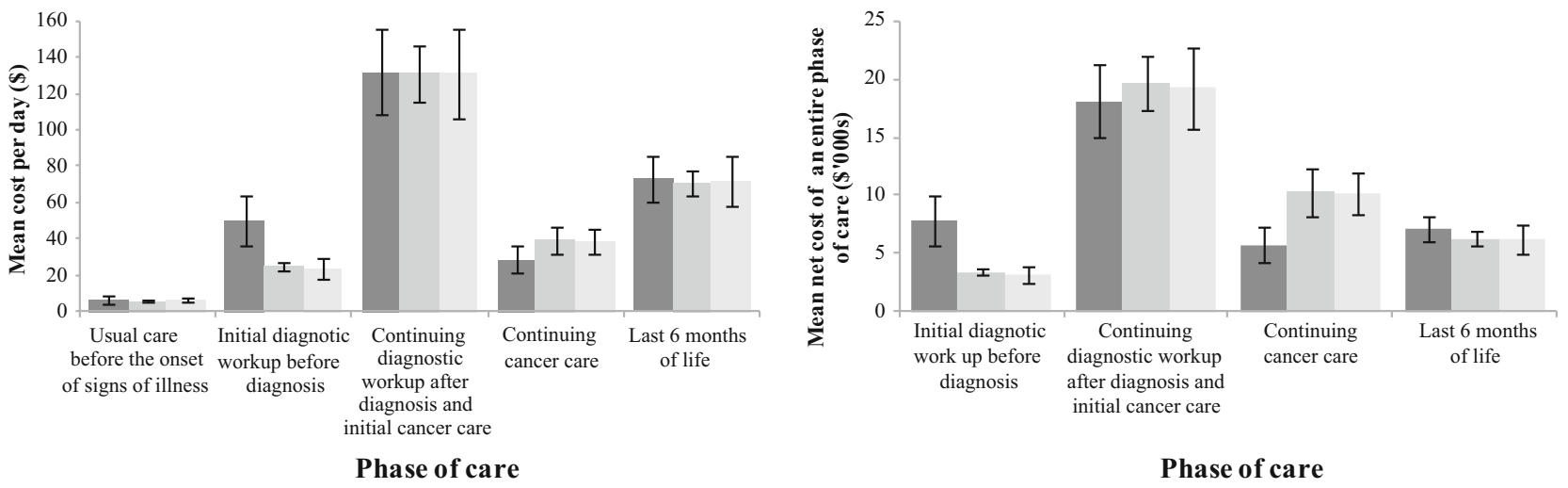

Patients with CUP ( $n=395)$

Patients with CKP $(\mathrm{n}=5,444)$

Matched patients with CKP $(\mathrm{n}=395)$

Fig. 2 continued

associated with higher risk of death and appeared to account for most of the observed $31 \%$ increase in risk of mortality for patients with CUP. This association should be interpreted with caution as it might also be influenced by unknown differences in tumour biologic behaviour, disease burden and/or the functional status of patients between the CUP and matched CKP groups [45]. However, no evidence exists yet showing CUP tumours harbour distinct genetic traits compared with CKP tumours [6, 46]. Thus, it might be reasonable to infer that many patients were considered unsuitable for more specific targeted cancer treatments and were treated with more intensive empiric cytotoxic chemotherapy due to the absence of knowledge about their primary tumour site. Therefore, these data suggest that identification of the primary tumour site early in the course of metastatic disease may enable greater precision of cancer therapy and potentially improve patients' survival by allowing patients with CUP to benefit more from available healthcare resources during the "continuing cancer care" phase, similar to their counterparts with CKP [29].
Currently, a precision medicine approach can be applied to the treatment of many metastatic cancers, including cetuximab and panitumumab in colorectal cancer [27], rituximab in lymphoma [47], bevacizumab in renal cell carcinoma [48] and colorectal cancer [49], trastuzumab in breast cancer [50], erlotinib in lung cancer [51] and sorafenib and sunitinib in renal cell carcinoma [52]. Knowledge of the biologic characteristics of an individual tumour (e.g. somatic EGFR, KRAS, ALK, HER2, BRAF, CD2O, and $R O S 1$ gene status) is increasingly valuable in the clinical management and treatment selection for patients with metastatic cancer [27, 47-52]. However, the ability to identify a primary tumour site is also increasingly important and likely to remain important for the foreseeable future [8]. For instance, knowledge of the primary tumour site is needed to interpret somatic mutation results. Information about KRAS mutation status has quite different implications depending on whether the primary site is lung versus colon [53]. Similarly, information regarding hormone receptor status has quite different implications depending on whether the primary tumour site is breast or 
ovary. Generally, targeted therapies are studied, approved and reimbursed solely within the context of an identified primary tumour site [27, 47-52]. Furthermore, finding the primary tumour site might prevent local complications (e.g. bleeding from the primary tumour, obstruction or perforation of a viscus), give a better guide to prognosis and help integrate patient-centred care into practice by helping the physician communicate with the patient, who may also find value in knowing where their cancer originated from, independent of effects on prognosis and treatment $[6,46]$. Therefore, information about the primary tumour site and its inherent biologic characteristics are both valuable and complementary in precision management of patients with CUP $[6,46]$.

In our study, having CUP was associated with a significant increase in the mean net cost of overall healthcare utilization during the "initial diagnostic workup before diagnosis" phase. Patients with CUP were also more likely than those with CKP to receive several diagnostic tests and procedures during the diagnostic workup. These findings indicate that patients with CUP underwent more extensive diagnostic investigations and incurred more costs to the healthcare system related to difficulties in diagnosis. Despite this, they remained without a primary tumour classification following their clinical and pathologic diagnostic workup, according to our study of real-life Canadian clinical practice. Although data about IHC investigation were not available and thus our study was unable to investigate pathological diagnostic barriers in these patients, current Canadian clinical practice has not been influenced by the availability of accurate gene expression profiling assays to help identify the primary tumour in metastatic cancer $[1,10,54]$. Our data suggest the need for gene expression profiling techniques to complement current traditional diagnostic procedures (e.g. IHC analyses, endoscopies, CT scans, X-rays, MRI scans, etc.) when dealing with diagnostic difficulties so that the primary tumour can be classified early in the course of metastatic disease. This may allow patients to avoid unnecessary or even painful and distressing extensive investigations and the healthcare system to avoid unnecessary costs. Recently, the use of a gene expression profiling test to aid in identifying the primary tumour when current clinical and pathological diagnostic evaluation failed to provide a diagnosis of primary tumour site in CUP patients was found to be cost effective from the Canadian healthcare system perspective [34]. Our data further suggest some value may be gained from using gene expression profiling techniques at earlier stages in the diagnostic workup.

Although this is a retrospective cohort study and our results must be interpreted with caution, the incidence of
CUP and the impact of primary tumour classification on therapy, patient outcomes and cost of care throughout the course of metastatic disease are unlikely to be studied in prospective designed analyses or randomized controlled trials. This is because CUP syndrome is extremely heterogeneous and the ultimate study design would require a very large sample size. Randomization to either sitespecific therapy or standard CUP empiric therapy following primary tumour classification also may not be ethical because it is already believed that, for many primary tumour diagnoses, the standard empiric therapy for CUP would be less effective. Our retrospective cohort study is an example of an alternative approach.

This study used rigorous linkage of high-quality population data from comprehensive heath administrative databases and yielded a true incidence rate of CUP given our strict inclusion criteria of histologically confirmed metastatic disease and at least 6-month survival. We took special care to avoid sources of bias and confounding in our study by conducting a matched cohort analysis where the matched group of patients with CKP clearly had the same underlying population of Manitoba as patients with CUP and were matched on all known patient and tumour characteristics. In fact, the smaller number of patients included in our matched cohort analysis compared with our overall cohort permits future investigation of more detailed and risk factors of having a CUP. For instance, important factors associated with diagnostic workup obtained from detailed medical histories or biologic markers such as specialist referrals and type and frequency of immunohistochemistry tests (i.e. information not collected by the databases used for this study) become feasible to investigate in order to understand the actual diagnostic barriers in patients with CUP. Future studies can further link such real-life cases with their specimens from banks of tumour tissue samples to study the potential utility of personalized diagnostic technologies such as the newly developed gene expression profiling assays.

In addition, the diagnostic and prognostic parameters and phase-specific cost estimates described in our study represent key imputes necessary to build decision analytic models designed to evaluate the cost effectiveness of such personalized diagnostic technologies in CUP. Developing these data is important to formulate new provincial guidelines and reimbursement policies for CUP management and is necessary to inform healthcare resource allocation and fiscal planning for the prospective precision medicine era of care for CUP. Thus, our data will ultimately contribute in developing additional clinical evidence to optimize a precision medicine strategy as a standard of care for patients with CUP. 


\section{Conclusion}

CUP is the fifth most common cancer type diagnosed in patients presenting with metastatic cancer and is more common in women than in men. Compared with CKP patients, CUP patients receive fewer site-specific and targeted therapies but more empiric therapies, have reduced OS, and use more healthcare resources for diagnostic workup but less healthcare resources for cancer care. Identifying the primary tumour in CUP patients might enable the use of more precise anticancer therapy with the goals of improving OS and more efficiently allocating healthcare resources.

Acknowledgements The authors thank the Department of Epidemiology and Cancer Registry of CCMB and Manitoba Health, Healthy Living and Seniors for its support throughout the study. The results and conclusions are those of the authors, and no official endorsement by Manitoba Health, Healthy Living and Seniors is intended or should be inferred.

Data Availability Statement The datasets generated and/or analysed during the current study are not publicly available according to privacy rules of the MCR and Manitoba Health.

Author Contributions MBH and GSZ contributed to the study design, data collection, data analyses, data interpretation and writing. EW, MB, GR, SS and PKR contributed to the study design, data interpretation, and writing. SMM contributed to the study design, data collection, data interpretation and writing. JSH contributed to the study design and writing.

\section{Compliance with Ethical Standards}

Conflict of interest MBH, EW, SMM, MB, GR, SS, PKR, JSH and GSZ declare no potential conflicts of interest.

Funding This work was supported by the Canadian Institutes of Health Research (CIHR) (operating Grant \#231890; GSZ [PI]); the CIHR Strategic Training Program in Cancer Research and Technology Transfer (CaRTT) and Academic Development Grant from Western University to MBH; the Canada Research Chairs program to GSZ, PKR and SMM; and the Great-West Life, London Life and Canada Life Junior Investigator of the Canadian Cancer Society (Grant \# 2011-700644) to SMM. Data collection and analyses were approved by the University of Manitoba Health Research Ethics Board, Manitoba Health Information Privacy Committee and Western University Health Research Ethics Board.

Open Access This article is distributed under the terms of the Creative Commons Attribution-NonCommercial 4.0 International License (http://creativecommons.org/licenses/by-nc/4.0/), which permits any noncommercial use, distribution, and reproduction in any medium, provided you give appropriate credit to the original author(s) and the source, provide a link to the Creative Commons license, and indicate if changes were made.

\section{References}

1. Oien KA, Dennis JL. Diagnostic work-up of carcinoma of unknown primary: from immunohistochemistry to molecular profiling. Ann Oncol. 2012;23(Suppl 10):x271-7.

2. BC Cancer Agency. Cancer management guidelines. 2016. http:// www.bccancer.bc.ca/HPI/CancerManagementGuidelines/default. htm. Accessed 18 Aug 2016.

3. Stella GM, Senetta R, Cassenti A, Ronco M, Cassoni P. Cancers of unknown primary origin: current perspectives and future therapeutic strategies. J Transl Med. 2012;10:12.

4. Briasoulis E, Pavlidis N. Cancer of unknown primary origin. Oncologist. 1997;2(3):142-52.

5. Levine MN, Drummond MF, Labelle RJ. Cost-effectiveness in the diagnosis and treatment of carcinoma of unknown primary origin. CMAJ. 1985;133(10):977-87.

6. Greco FA, Oien K, Erlander M, Osborne R, Varadhachary G, Bridgewater $\mathrm{J}$, et al. Cancer of unknown primary: progress in the search for improved and rapid diagnosis leading toward superior patient outcomes. Ann Oncol. 2012;23(2):298-304.

7. Dumur CI, Lyons-Weiler M, Sciulli C, Garrett CT, Schrijver I, Holley TK, et al. Interlaboratory performance of a microarraybased gene expression test to determine tissue of origin in poorly differentiated and undifferentiated cancers. J Mol Diagn. 2008;10(1):67-77.

8. Chiang WM, Kapadia M, Laver NV, Nystrom JS. Cancer of unknown primary: from immunohistochemistry to gene expression profiling. J Clin Oncol. 2012;30(29):e300-2.

9. Greco FA, Spigel DR, Yardley DA, Erlander MG, Ma XJ, Hainsworth JD. Molecular profiling in unknown primary cancer: accuracy of tissue of origin prediction. Oncologist. 2010;15(5):500-6.

10. Greco FA. Cancer of unknown primary site: improved patient management with molecular and immunohistochemical diagnosis. Am Soc Clin Oncol Educ Book. 2013:175-81. https://doi.org/ 10.1200/EdBook_AM.2013.33.175.

11. Morris GJ, Greco FA, Hainsworth JD, Engstrom PF, Scialla S, Jordan WE 3rd, et al. Cancer of unknown primary site. Semin Oncol. 2010;37(2):71-9.

12. Greco FA. Therapy of adenocarcinoma of unknown primary: are we making progress? J Natl Compr Cancer Netw. 2008;6(10):1061-7.

13. Varadhachary GR, Talantov D, Raber MN, Meng C, Hess KR, Jatkoe T, et al. Molecular profiling of carcinoma of unknown primary and correlation with clinical evaluation. J Clin Oncol. 2008;26(27):4442-8.

14. Bloom G, Yang IV, Boulware D, Kwong KY, Coppola D, Eschrich S, et al. Multi-platform, multi-site, microarray-based human tumor classification. Am J Pathol. 2004;164(1):9-16.

15. Bridgewater J, van Laar R, Floore A, Van TVL. Gene expression profiling may improve diagnosis in patients with carcinoma of unknown primary. Br J Cancer. 2008;98(8):1425-30.

16. Buckhaults P, Zhang Z, Chen YC, Wang TL, St Croix B, Saha S, et al. Identifying tumor origin using a gene expression-based classification map. Cancer Res. 2003;63(14):4144-9.

17. Horlings HM, van Laar RK, Kerst JM, Helgason HH, Wesseling $\mathrm{J}$, van der Hoeven JJ, et al. Gene expression profiling to identify the histogenetic origin of metastatic adenocarcinomas of unknown primary. J Clin Oncol. 2008;26(27):4435-41.

18. Ma XJ, Patel R, Wang X, Salunga R, Murage J, Desai R, et al. Molecular classification of human cancers using a 92-gene real- 
time quantitative polymerase chain reaction assay. Arch Pathol Lab Med. 2006;130(4):465-73.

19. Monzon FA, Lyons-Weiler M, Buturovic LJ, Rigl CT, Henner WD, Sciulli C, et al. Multicenter validation of a 1,550-gene expression profile for identification of tumor tissue of origin. J Clin Oncol. 2009;27(15):2503-8.

20. Monzon FA, Koen TJ. Diagnosis of metastatic neoplasms: molecular approaches for identification of tissue of origin. Arch Pathol Lab Med. 2010;134(2):216-24.

21. Rosenfeld N, Aharonov R, Meiri E, Rosenwald S, Spector Y, Zepeniuk M, et al. MicroRNAs accurately identify cancer tissue origin. Nat Biotechnol. 2008;26(4):462-9.

22. Su AI, Welsh JB, Sapinoso LM, Kern SG, Dimitrov P, Lapp H, et al. Molecular classification of human carcinomas by use of gene expression signatures. Cancer Res. 2001;61(20):7388-93.

23. Talantov D, Baden J, Jatkoe T, Hahn K, Yu J, Rajpurohit Y, et al. A quantitative reverse transcriptase-polymerase chain reaction assay to identify metastatic carcinoma tissue of origin. J Mol Diagn. 2006;8(3):320-9.

24. van Laar RK, Ma XJ, de Jong D, Wehkamp D, Floore AN, Warmoes MO, et al. Implementation of a novel microarray-based diagnostic test for cancer of unknown primary. Int $\mathbf{J}$ Cancer. 2009;125(6):1390-7.

25. Rosenwald S, Gilad S, Benjamin S, Lebanony D, Dromi N, Faerman A, et al. Validation of a microRNA-based qRT-PCR test for accurate identification of tumor tissue origin. Mod Pathol. 2010;23(6):814-23.

26. Pillai R, Deeter R, Rigl CT, Nystrom JS, Miller MH, Buturovic L, et al. Validation and reproducibility of a microarray-based gene expression test for tumor identification in formalin-fixed, paraffin-embedded specimens. J Mol Diagn. 2011;13(1):48-56.

27. Chhatrala R, Thanavala Y, Iyer R. Targeted therapy in gastrointestinal malignancies. J Carcinog. 2014;13:4.

28. Abu-Rustum N, Chi D, Coleman RL, del Campo JM, Fotopoulou C, Frumovitz M, et al. Summary of the 2014 MD Anderson International Meeting in Gynecologic Oncology: emerging therapies in gynecologic cancer. Gynecol Oncol. 2014;134(1):6-9.

29. Hainsworth JD, Rubin MS, Spigel DR, Boccia RV, Raby S, Quinn R, et al. Molecular gene expression profiling to predict the tissue of origin and direct site-specific therapy in patients with carcinoma of unknown primary site: a prospective trial of the Sarah Cannon research institute. J Clin Oncol. 2013;31(2):217-23.

30. Hannouf M, Zaric GS. Cost-effectiveness analysis using registry and administrative data. Operations research and health care policy. Int Ser Oper Res Manag Sci. 2013;190:341-61.

31. Hannouf MB, Brackstone M, Xie B, Zaric GS. Evaluating the efficacy of current clinical practice of adjuvant chemotherapy in postmenopausal women with early-stage, estrogen or progesterone receptor-positive, one-to-three positive axillary lymph node, breast cancer. Curr Oncol. 2012;19(5):e319-28.

32. Hannouf MB, Xie B, Brackstone M, Zaric GS. Cost effectiveness of a 21-gene recurrence score assay versus Canadian clinical practice in post-menopausal women with early-stage estrogen or progesterone-receptor-positive, axillary lymph-node positive breast cancer. Pharmacoeconomics. 2014;32(2):135-47.

33. Hannouf MB, Xie B, Brackstone M, Zaric GS. Cost-effectiveness of a 21-gene recurrence score assay versus Canadian clinical practice in women with early-stage estrogen- or progesteronereceptor-positive, axillary lymph-node negative breast cancer. BMC Cancer. 2012;02(12):447.

34. Hannouf MB, Winquist E, Mahmud SM, Brackstone M, Sarma S, Rodrigues G, Rogan P, Hoch JS, Zaric GS. Cost-effectiveness of using a gene expression profiling test to aid in identifying the primary tumour in patients with cancer of unknown primary. Pharmacogenom J. 2017;17(3):286-300.
35. Finlayson G, Reimer J, Dahl M, Stargardter M, McGowan K. The direct cost of hospitalizations in Manitoba, 2005/06. Winnipeg: Manitoba Centre for Health Policy; 2009.

36. Finlayson G, Nowicki D, Roos NP, Shanahan M, Black C. Hospital case-mix costing project: using the Manitoba management information system: a first step. Winnipeg: Manitoba Centre for Health Policy and Evaluation; 1999.

37. Bank of Canada. Home $>$ rates and statistics $>$ related information $>$ inflation calculator [Web resource]. Ottawa, ON: Bank of Canada. http://www.bankofcanada.ca/en/rates/inflation_calc. html. Accessed 20 June 2016.

38. Yabroff KR, Lamont EB, Mariotto A, Warren JL, Topor M, Meekins A, et al. Cost of care for elderly cancer patients in the United States. J Natl Cancer Inst. 2008;100(9):630-41.

39. Mittmann N, Liu N, Porter J, Seung SJ, Isogai PK, Saskin R, et al. Utilization and costs of home care for patients with colorectal cancer: a population-based study. CMAJ Open. 2014;2(1):E11-7.

40. Cipriano LE, Romanus D, Earle CC, Neville BA, Halpern EF, Gazelle GS, et al. Lung cancer treatment costs, including patient responsibility, by disease stage and treatment modality, 1992 to 2003. Value Health. 2011;14(1):41-52.

41. McCarron CE, Ernst S, Cao JQ, Zaric GS. Population-based estimates of survival and cost for metastatic melanoma. Curr Oncol. 2015;22(5):326-32.

42. Austin PC. An introduction to propensity score methods for reducing the effects of confounding in observational studies. Multivar Behav Res. 2011;46(3):399-424.

43. Stuart EA. Matching methods for causal inference: a review and a look forward. Stat Sci. 2010;25(1):1-21.

44. Schafer JL, Kang J. Average causal effects from nonrandomized studies: a practical guide and simulated example. Psychol Methods. 2008;13(4):279-313.

45. Pentheroudakis G, Greco FA, Pavlidis N. Molecular assignment of tissue of origin in cancer of unknown primary may not predict response to therapy or outcome: a systematic literature review. Cancer Treat Rev. 2009;35(3):221-7.

46. Greco FA. Cancer of unknown primary site: still an entity, a biological mystery and a metastatic model. Nat Rev Cancer. 2014;14(1):3-4.

47. Coiffier B. Rituximab therapy in malignant lymphoma. Oncogene. 2007;26(25):3603-13.

48. Escudier B, Pluzanska A, Koralewski P, Ravaud A, Bracarda S, Szczylik C, et al. Bevacizumab plus interferon alfa-2a for treatment of metastatic renal cell carcinoma: a randomised, doubleblind phase III trial. Lancet. 2007;370(9605):2103-11.

49. Hurwitz H, Fehrenbacher L, Novotny W, Cartwright T, Hainsworth $\mathrm{J}$, Heim $\mathrm{W}$, et al. Bevacizumab plus irinotecan, fluorouracil, and leucovorin for metastatic colorectal cancer. N Engl J Med. 2004;350(23):2335-42.

50. Hudis CA. Trastuzumab-mechanism of action and use in clinical practice. N Engl J Med. 2007;357(1):39-51.

51. Tsao MS, Sakurada A, Cutz JC, Zhu CQ, Kamel-Reid S, Squire J, et al. Erlotinib in lung cancer-molecular and clinical predictors of outcome. N Engl J Med. 2005;353(2):133-44.

52. Brugarolas J. Renal-cell carcinoma-molecular pathways and therapies. N Engl J Med. 2007;356(2):185-7.

53. Hannouf MB, Winquist E, Mahmud SM, Brackstone M, Sarma S, Rodrigues G, Rogan P, Hoch JS, Zaric GS. The clinical significance of occult gastrointestinal primary tumours in metastatic cancer: a population retrospective cohort study. Cancer Res Treat. 2017. doi:10.4143/crt.2016.532.

54. Greco FA. Molecular diagnosis of the tissue of origin in cancer of unknown primary site: useful in patient management. Curr Treat Options Oncol. 2013;14(4):634-42. 